\title{
A case of renovascular hypertension with incidental primary bilateral macronodular adrenocortical hyperplasia
}

\author{
Takuya Higashitani(D1,*, Shigehiro Karashima1,*, Daisuke Aono1, Seigoh Konishi1,2, Mitsuhiro Kometani1, Rie Oka1, \\ Masashi Demura3 ${ }^{3}$ Kenji Furukawa4, Yuto Yamazaki ${ }^{5}$, Hironobu Sasano5, Takashi Yoneda ${ }^{1,6}$ and Yoshiyu Takeda ${ }^{1}$
}

1Division of Endocrinology and Hypertension, Department of Cardiovascular and Internal Medicine, Graduate School of Medical Science, Kanazawa University, Kanazawa, Ishikawa, Japan, 2Department of Internal Medicine, Keiju Medical Center, Nanao, Ishikawa, Japan, ${ }^{3}$ Department of Hygiene, Graduate School of Medical Science, Kanazawa University, Kanazawa, Ishikawa, Japan, ${ }^{4 H e a l t h}$ Care Center, Japan Advanced Institute of Science and Technology, Nomi, Ishikawa, Japan, 5Department of Pathology, Tohoku University Hospital, Sendai, Miyagi, Japan, and ${ }^{6}$ Department of Health Promotion and Medicine of the Future, Kanazawa University, Kanazawa, Ishikawa, Japan

Correspondence should be addressed to $Y$ Takeda

Email

takeday@med.kanazawa-u. ac.jp

\section{Summary}

Renovascular hypertension (RVHT) is an important and potentially treatable form of resistant hypertension. Hypercortisolemia could also cause hypertension and diabetes mellitus. We experienced a case wherein adrenalectomy markedly improved blood pressure and plasma glucose levels in a patient with RVHT and low-level autonomous cortisol secretion. A 62-year-old Japanese man had been treated for hypertension and diabetes mellitus for 10 years. He was hospitalized because of a disturbance in consciousness. His blood pressure (BP) was 236/118 mmHg, pulse rate was 132 beats/min, and plasma glucose level was $712 \mathrm{mg} / \mathrm{dL}$. Abdominal CT scanning revealed the presence of bilateral adrenal masses and left atrophic kidney. Abdominal magnetic resonance angiography demonstrated marked stenosis of the left main renal artery. The patient was subsequently diagnosed with atherosclerotic RVHT with left renal artery stenosis. His left adrenal lobular mass was over $40 \mathrm{~mm}$ and it was clinically suspected the potential for cortisol overproduction. Therefore, laparoscopic left nephrectomy and adrenalectomy were simultaneously performed, resulting in improved $\mathrm{BP}$ and glucose levels. Pathological studies revealed the presence of multiple cortisol-producing adrenal nodules and aldosterone-producing cell clusters in the adjacent left adrenal cortex. In the present case, the activated renin-angiotensinaldosterone system and cortisol overproduction resulted in severe hypertension, which was managed with simultaneous unilateral nephrectomy and adrenalectomy.

\section{Learning points:}

- Concomitant activation of the renin-angiotensin-aldosterone system and cortisol overproduction may contribute to the development of severe hypertension and lead to lethal cardiovascular complications.

- Treatment with simultaneous unilateral nephrectomy and adrenalectomy markedly improves BP and blood glucose levels.

- CYP11B2 immunohistochemistry staining revealed the existence of aldosterone-producing cell clusters (APCCs) in the adjacent non-nodular adrenal gland, suggesting that APCCs may contribute to aldosterone overproduction in patients with RVHT. 


\section{Background}

Activation of the renin-angiotensin-aldosterone system (RAAS) increases systemic blood pressure (BP). Renovascular hypertension (RVHT) is one of the most common types of secondary hypertension. RVHT causes hyperreninemic hyperaldosteronism and that is reported to affect $5 \%$ of the adult hypertensive population (1).

Low-level autonomous cortisol secretion is a condition characterized by hypercortisolism in the absence of physical signs of specific apparent cortisol excess. Lowlevel autonomous cortisol secretion is also associated with an increased risk of developing hypertension, diabetes, and dyslipidemia (2). However, very few cases of concomitant RVHT and low-level autonomous cortisol secretion have been reported in the literature. Activation of the RAAS and cortisol overproduction could both contribute to the development of severe hypertension and, finally, to lethal cardiovascular complications. To our knowledge, this is the first case report of RVHT with cortisol-producing adrenal masses.

\section{Case presentation}

A 62-year-old Japanese patient was treated for diabetes, hypertension, and dyslipidemia for 10 years. He was found unconscious and was admitted to a hospital.

\section{Investigation}

On presentation, his $\mathrm{BP}$ was $236 / 118 \mathrm{mmHg}$ and his pulse rate was 132 beats $/ \mathrm{min}$. His BMI was $21.0 \mathrm{~kg} / \mathrm{m}^{2}$. His plasma glucose level was $712 \mathrm{mg} / \mathrm{dL}$ and urinary ketone bodies were not detected. His blood $\mathrm{pH}$ was 7.273 and the calculated plasma osmotic pressure was $320 \mathrm{mosmol} / \mathrm{L}$. His neck was supple, and his lungs were clear to auscultation, no heart murmurs. No physical features of Cushing's syndrome were observed, and abdominal bruits were inaudible. Cranial MRI revealed multiple high-signal areas on T2-weighted and fluid-attenuated inversion recovery images, suggesting posterior reversible encephalopathy syndrome. The patient experienced hypertensive emergency and was diagnosed with hyperosmolar hyperglycemic nonketotic syndrome. He was treated with i.v. antihypertensive agents and insulin. BP and glucose levels improved, and he regained consciousness. Intravenous antihypertensive agents were changed to oral agents after 3 days, his 24-h BP showed dipping pattern but mean BP remained high (mean 171/89 mmHg) during ambulatory BP monitoring.

His biochemical and hormonal data are shown in Table 1. His plasma renin activity (PRA) was 10.7 (normal range: $0.2-2.7 \mathrm{ng} / \mathrm{mL} / \mathrm{h}$ ) and plasma aldosterone concentration (PAC) was 173 (normal range: 20-30 pg/mL). He was in the state of hyperreninemic hyperaldosteronism and DHEA-S was 109 (normal range: 24-244 $\mu \mathrm{g} / \mathrm{dL}$ ). Abdominal CT revealed a $45-\mathrm{mm}$ leftsided lobular adrenal mass (unenhanced Hounsfield unit (HU):14.2) with microcalcifications, a 20-mm right-sided adrenal mass (unenhanced HU: 11.3), and left atrophic kidney (Fig. 1A, B and C). Adrenal chemical shift MRI showed lower signal intensity in the left adrenal mass on the opposed-phase image compared with the in-phase image. The captopril challenge test demonstrated that the PRA increased from $16.5 \mathrm{ng} / \mathrm{mL} / \mathrm{h}$ to $61.1 \mathrm{ng} / \mathrm{mL} / \mathrm{h}$ after loading $50 \mathrm{mg}$ of captopril over $60 \mathrm{~min}$. Abdominal MR angiography revealed severe stenosis of the left main artery (Fig. 1D). Selective renal venous sampling indicated a left/right renal venous PRA ratio of 5.5 (left kidney: $57 \mathrm{ng} / \mathrm{mL} / \mathrm{h}$ vs right kidney: $11 \mathrm{ng} / \mathrm{mL} / \mathrm{h}$ ), suggesting that renin secretion was greater on the left side. Table 2 demonstrates the results of the adrenal venous sampling with or without adrenocorticotropic hormone (ACTH) stimulation. The findings indicated hypersecretion of aldosterone from the bilateral adrenal gland.

Serum cortisol levels decreased to only $3.1 \mu \mathrm{g} / \mathrm{dL}$ and $2.2 \mu \mathrm{g} / \mathrm{dL}$ post the dexamethasone $1-\mathrm{mg}$ and $8-\mathrm{mg}$ suppression tests, respectively, suggesting the overproduction of cortisol. ${ }^{131}$ I-adosterol scintigraphy showed high uptake in the left adrenal gland. The uptake in the right adrenal gland was not suppressed. ${ }^{18} \mathrm{~F}-\mathrm{FDG}$ PET scan showed mild uptake of radioactivity at the left adrenal gland and no uptake at the right adrenal gland. Pheochromocytoma was ruled out because urinary metanephrine excretion was within the normal range, and ${ }^{123}$ I-MIBG scintigraphy findings were negative for pheochromocytoma.

\section{Treatment}

The patient was diagnosed with RVHT due to left renal artery stenosis, and given the higher radiographic uptake on the left side, a left adrenalectomy was favored in keeping with existing guidelines (2). Laparoscopic left nephrectomy and left adrenalectomy were simultaneously performed. 
Table 1 Laboratory investigations, 24-h ambulatory blood pressure monitoring, and renal function parameters before and after operation.

\begin{tabular}{|c|c|c|c|}
\hline Variable & Normal range & Before & After \\
\hline \multicolumn{4}{|l|}{ Biochemical tests } \\
\hline Serum potassium (mEq/L) & $3.5-4.9$ & 3.7 & 4.7 \\
\hline Serum creatinine $(\mathrm{mg} / \mathrm{dL})$ & $0.60-1.00$ & 2.97 & 2.42 \\
\hline Urine protein excretion (g/day) & $0.02-0.06$ & 5.5 & 3.4 \\
\hline Fasting plasma glucose (mg/dL) & 69-109 & 152 & 106 \\
\hline Fasting plasma insulin $(\mu \mathrm{U} / \mathrm{mL})$ & $2.2-12.4$ & 6.4 & 10.9 \\
\hline HOMA-R & $<1.6$ & 2.4 & 1.7 \\
\hline Urine C-peptide excretion ( $\mu$ g/day) & $17-181$ & 29.2 & 62.7 \\
\hline $\mathrm{HbA} 1 \mathrm{c}(\%)$ & $4.6-6.2$ & 10.0 & 6.4 \\
\hline Total cholesterol (mg/dL) & $128-219$ & 240 & 144 \\
\hline HDL cholesterol (mg/dL) & 40-99 & 30 & 50 \\
\hline LDL cholesterol (mg/dL) & $57-139$ & 98 & 75 \\
\hline Triglyceride (mg/dL) & $30-149$ & 562 & 93 \\
\hline \multicolumn{4}{|l|}{ Renin-angiotensin-aldosterone system } \\
\hline PRA (ng/mL/h)/PAC (pg/mL) & $0.2-2.7 / 20-130$ & $10.7 / 173$ & $2.4 / 34$ \\
\hline PRA (ng/mL/h)/PAC (pg/mL) at CCT baseline & & $16.5 / 480$ & $1.1 / 123$ \\
\hline PRA (ng/mL/h)/PAC (pg/mL) at CCT after $60 \mathrm{~min}$ & & $54.3 / 271$ & $1.7 / 109$ \\
\hline Urine collection aldosterone ( $\mu \mathrm{g} /$ day) & $<10$ & 10.9 & 5.5 \\
\hline \multicolumn{4}{|l|}{ HPA axis } \\
\hline ACTH (pg/mL)/cortisol ( $\mu \mathrm{g} / \mathrm{dL})$ at 8:00 & $<46 / 6.2-19.4$ & $15.9 / 15.8$ & $75.8 / 11.1$ \\
\hline ACTH $(\mathrm{pg} / \mathrm{mL}) /$ cortisol $(\mathrm{pg} / \mathrm{mL})$ at 23:00 & & 23.2/4.6 & $26.1 / 4.8$ \\
\hline ACTH $(\mathrm{pg} / \mathrm{mL}) /$ cortisol $(\mu \mathrm{g} / \mathrm{mL})$ after $1 \mathrm{mg}$ DEX & & $2.4 / 3.1$ & $<5.0 / 1.2$ \\
\hline 24-h urine cortisol ( $\mu \mathrm{g} /$ day) & $11.2-80.3$ & 58.7 & 15.1 \\
\hline \multicolumn{4}{|l|}{ 24-h ambulatory blood pressure monitoring } \\
\hline 24-h mean SBP/DBP (mmHg) & & $171 / 89$ & $147 / 82$ \\
\hline Daytime mean SBP/DBP (mmHg) & & $179 / 92$ & $152 / 85$ \\
\hline Night-time SBP/DBP (mmHg) & & $156 / 82$ & $135 / 75$ \\
\hline \multicolumn{4}{|l|}{ 93mTc-DTPA renography/renoscintigraphy } \\
\hline Right/left GFR (mL/min) & & $14.0 / 3.8$ & $17.2 /-$ \\
\hline
\end{tabular}

Medicines for hypertension, diabetes, and dyslipidemia before the nephrectomy and adrenalectomy: doxazosin 4 mg, nifedipine 40 mg, linagliptin 5 mg, repaglinide $1.5 \mathrm{mg}$, insulin aspart $14 \mathrm{U} / \mathrm{day}$, and insulin degludec $4 \mathrm{U} / \mathrm{day}$; Medicines, postoperatively: nifedipine CR $40 \mathrm{mg}$, repaglinide $1.5 \mathrm{mg}$. atorvastatin $10 \mathrm{mg}$, and tocopherol $600 \mathrm{mg}$.

ACTH, adrenocorticotropic hormone; CCT, captopril challenge test; DBP, diastolic blood pressure; DEX, dexamethasone; DTPA, diethylenetriaminepentaacetic acid; eGFR, Estimated glomerular filtration rate; HbA1c, hemoglobin A1c; HOMA-R, Homeostasis model assessment insulin resistance index; HPA, hypothalamic-pituitary-adrenal; PAC, plasma aldosterone concentration; PRA, plasma renin activity; SBP, systolic blood pressure.

\section{Outcome and follow-up}

The resected adrenal gland measured $50 \times 30 \times 15 \mathrm{~mm}$ and harbored multiple golden yellow-colored nodules without any foci of hemorrhage and/or necrosis (Fig. 2A and B). These nodules measured 2-7 $\mathrm{mm}$ and they were consistent with macronodular hyperplasia of the zona glomerulosa. The representative histopathological findings of these adrenocortical nodules are illustrated in Fig. 2B, C, D, E, F, $\mathrm{G}$ and $\mathrm{H}$. The nodules were relatively well-circumscribed but not encapsulated and were mainly composed of clear cells (Fig. 2B). Immunohistochemical analysis for 11-beta-hydroxylase (CYP11B1) and aldosterone synthase (CYP11B2) was performed as reported previously (3). The nodules were immunoreactive for CYP11B1 but not CYP11B2. In the adjacent adrenal gland, several aldosterone-producing cell clusters (APCCs) that were immunohistochemically positive for CYP11B2, but negative for CYP11B1, were detected (Fig. 2H).

Postoperatively, the BP, PRA, and serum cortisol levels were improved. The HbA1c levels decreased from $10 \%$ to $6.4 \%$. Moreover, the serum creatinine and urinary protein levels decreased from $3.0 \mathrm{mg} / \mathrm{dL}$ to $2.4 \mathrm{mg} / \mathrm{dL}$ and from $5.5 \mathrm{~g} /$ day to $3.4 \mathrm{~g} /$ day, respectively (Table 1 ). As for the right-sided adrenal mass, we will conduct an annual follow-up with abdominal CT.

\section{Discussion}

The goal of RVHT treatment is to resolve systemic hypertension without compromising renal function. There are three modes of RVHT treatment: (1) administration of RAAS inhibitors, such as angiotensin- 
A

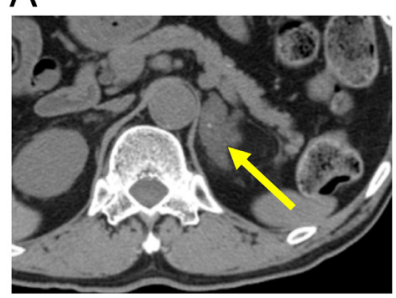

B

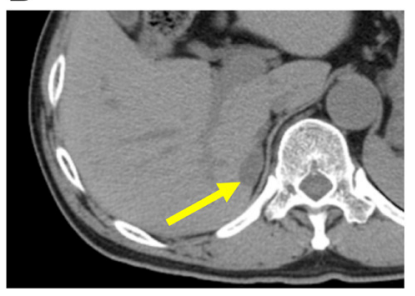

C
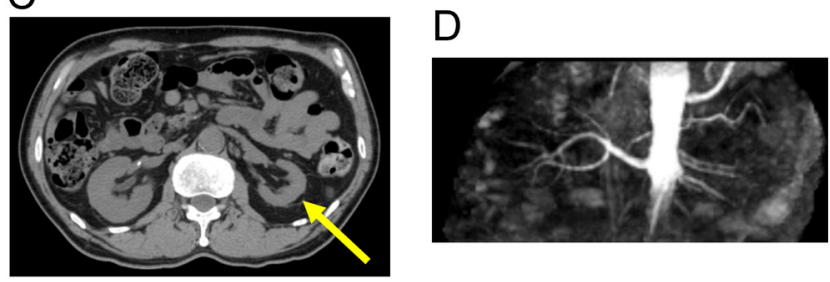

Figure 1

CT scanning and magnetic resonance angiography (MRA) findings prior to the surgery. CT scan showed a 45-mm left-sided lobular adrenal mass (arrow) (A), a 20-mm right-sided adrenal mass (arrow) (B), and a small left kidney (arrow) (C). MRA showed severe stenosis of the left main artery (D).

converting enzyme inhibitors and angiotensin II receptor blockers; (2) renal revascularization by percutaneous transluminal angioplasty or surgical revascularization; and (3) nephrectomy (4). In the present case, the glomerular filtration rate in the left kidney was not detectable with complete loss of left renal function. The use of RAAS inhibitors posed a challenge because of hyperkalemia and further aggravation of the patient's renal function. Renal revascularization is an invasive procedure and would not allow recovery of renal function because his left kidney had already ceased to function. The ACCF/AHA guidelines suggest that renal revascularization is not suitable for a nonfunctioning kidney, such as that

Table 2 Adrenal venous sampling with or without ACTH stimulation.

\begin{tabular}{|c|c|c|c|}
\hline & IVC & $\begin{array}{c}\text { Right adrenal } \\
\text { vein } \\
\end{array}$ & $\begin{array}{c}\text { Left adrenal } \\
\text { vein }\end{array}$ \\
\hline \multicolumn{4}{|c|}{ Without ACTH stimulation } \\
\hline PAC (pg/mL) & 136 & 5715 & 2389 \\
\hline Cortisol $(\mu \mathrm{g} / \mathrm{dL})$ & 5.7 & 29.2 & 17.9 \\
\hline PAC/Cortisol ratio & 23.9 & 195.7 & 133.5 \\
\hline \multicolumn{4}{|l|}{ With ACTH stimulation } \\
\hline PAC (pg/mL) & 407 & 39000 & 34000 \\
\hline Cortisol $(\mu \mathrm{g} / \mathrm{dL})$ & 18.5 & 504.0 & 740.0 \\
\hline PAC/Cortisol ratio & 22.0 & 77.4 & 45.9 \\
\hline
\end{tabular}

Blood samples were collected taken 30 min after bolus i.v. incection of ACTH (Tetracosactide) $0.25 \mathrm{mg}$ (25 IU) at ACTH-loading adrenal venous sampling.

ACTH, adrenocorticotropic hormone; PAC, plasma aldosterone concentration.

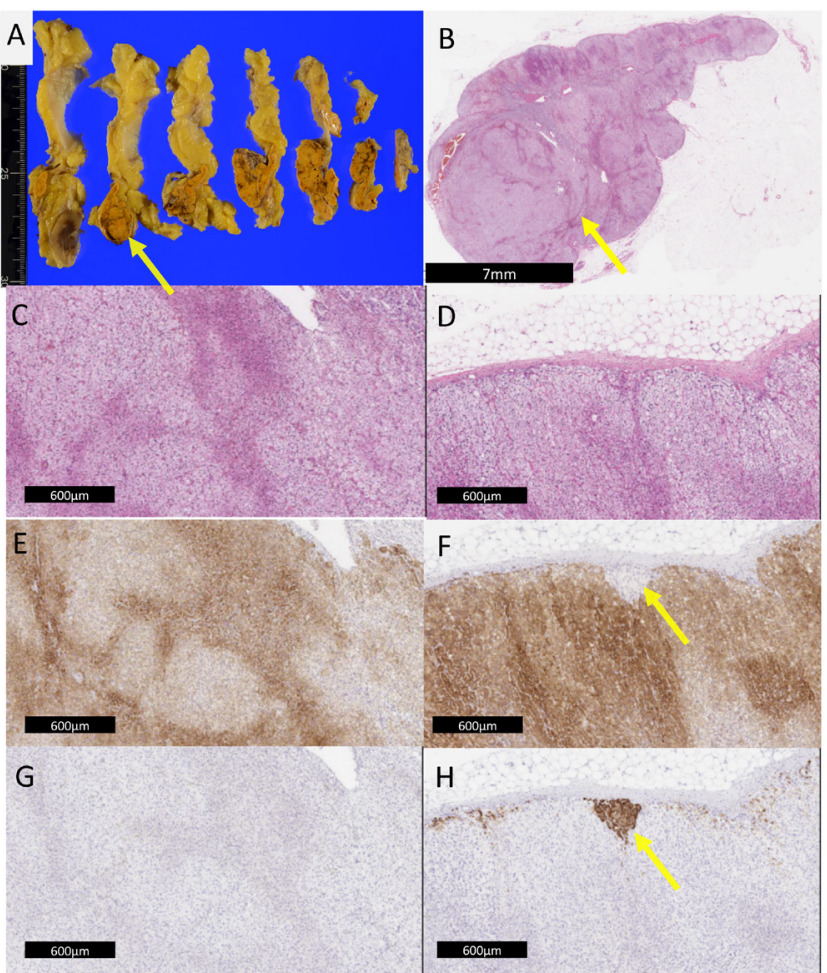

\section{Figure 2}

Histopathological characterization. Macroscopic view of the resected left adrenal gland (arrow) (A) and the specimen (arrow) (B). Hematoxylin-eosin staining and immunohistochemical staining for CYP11B1 and CYP11B2 in the adrenal nodules ( $C, E$, and $G$ ) and adjacent adrenal gland that includes APCCS. (arrow) (D, F, and H). ( $\times 40$ in original magnification).

in this case (5). Concerning nephrectomy, Lee et al. (6) reported that the mean reduction in the systolic BP was $27 \mathrm{mmHg}$ and that the diastolic BP was $17.5 \mathrm{mmHg}$ after laparoscopic unilateral nephrectomy for atrophic kidney in patients with RVHT. The patient's left adrenal lobular mass was over $40 \mathrm{~mm}$ and it was clinically suspected the potential for cortisol overproduction. Therefore, simultaneous combined laparoscopic nephrectomy and ipsilateral adrenalectomy were performed in this case. Tsunoda et al. (7) previously reported a rare case of concomitant aldosterone- and cortisol-co-secreting adrenal adenoma associated with RVHT in a 52-yearold man. Percutaneous transluminal renal angioplasty and laparoscopic left adrenalectomy were performed. Postoperatively, the BP decreased and both the PAC and PRA were normalized. The glomerular filtration rate was $24 \mathrm{~mL} / \mathrm{min}$ and $61 \mathrm{~mL} / \mathrm{min}$ on the diseased side and the healthy side, respectively. The residual renal function is an important factor to consider when selecting the treatment strategy for patients with RVHT.

In this case, the serum cortisol level post the 1-mg dexamethasone suppression test was $1.2 \mu \mathrm{g} / \mathrm{dL}$ after 
adrenalectomy. The HbA1c level and insulin resistance also improved after the surgery. An immunohistopathological evaluation revealed that the cortical cells in the nodules were diffusely positive for CYP11B1, which resembles the histological findings for primary bilateral macronodular adrenal hyperplasia (PBMAH). It has been shown that unilateral adrenalectomy improves glucose metabolism and insulin resistance in patients with Cushing's syndrome, including PBMAH or primary adrenocortical hyperplasia (7). Debillon et al. (8) also reported that diabetes was cured in four of six patients with PBMAH who underwent unilateral adrenalectomy. Thus, in the present case, an excessive glucocorticoid level was considered to contribute to insulin resistance.

The resected adrenal glands of patients with RVHT generally harbor hyperplasia of the zona glomerulosa. However, in this case, a remarkable CYP11B2 immunoreactivity was detected not diffusely as the hyperplasia of the zona glomerulosa but focally as APCCs in the adjacent adrenal cortex. APCCs were defined as CYP11B2-positive cell clusters within the zona glomerulosa involved in aldosterone biosynthesis (9). Previously, Nishimoto et al. (9) reported that APCCs were more frequently observed in normotensive patients with renal cell carcinoma than in patients with primary aldosteronism (PA), which is an endocrine disorder characterized by excessive secretion of the aldosterone hormone from the adrenal glands. Subsequently, Omata et al. (10) reported that APCCs were detected in nonhypertensive adrenal glands, with their number increasing with age, and that the number of APCCs further increased in patients with CT-negative PA. Sugiura et al. (11) also reported that APCCs could be the aldosteroneproducing lesions responsible for the development of PA. In the present case, immunohistochemistry staining for CYP11B2 revealed the existence of APCCs in the adjacent non-nodular adrenal gland, suggesting that APCCs may have contributed to the aldosterone overproduction in patients with RVHT. High renin activity might also play an important contribution to aldosterone production. Concerning the pathological examination of secondary hyperaldosteronism, immunohistological investigations are required for the clarification of other cases.

In summary, we describe the first case of RVHT associated with the presence of adrenal masses that have the potential for cortisol overproduction. Simultaneous laparoscopic nephrectomy and adrenalectomy ameliorated aldosterone and cortisol overproduction, resulting in improved BP and plasma glucose levels without impairing the renal function. Immunohistochemical positivity for
CYP11B2 puts forth APCCs as aldosterone-producing lesions responsible for the development of RVHT. However, the role of APCCs in RVHT pathology warrants further investigation.

\section{Declaration of interest}

The authors declare that there is no conflict of interest that could be perceived as prejudicing the impartiality of the research reported.

\section{Funding}

This research did not receive any specific grant from any funding agency in the public, commercial, or not-for-profit sector.

\section{Patient consent}

Written informed consent was obtained from the patient for publication of this case report and accompanying images.

\section{Author contribution statement}

$\mathrm{T} \mathrm{H}, \mathrm{S} \mathrm{K}, \mathrm{M} \mathrm{K}$ and $\mathrm{R} \mathrm{O}$ wrote the paper. $\mathrm{Y} \mathrm{Y}$ and $\mathrm{H} \mathrm{S}$ performed immunohistological analysis. D A, S K, and M D made figures and tables. $\mathrm{K} F, \mathrm{~T} Y$ and $\mathrm{Y} T$ supervised the case report. All authors reviewed the manuscript.

\section{Acknowledgements}

The authors thank Mikiya Usukura and Ayako Ishibashi-Wakayama for their advice regarding this research. The authors also thank Yukiko Takamiya and Chiyoko Takata for their secretarial assistance.

\section{References}

1 Derkx FH \& Schalekamp MA. Renal artery stenosis and hypertension. Lancet 1994344 237-239. (https://doi.org/10.1016/s01406736(94)93002-3)

2 Fassnacht M, Arlt W, Bancos I, Dralle H, Newell-Price J, Sahdev A, Tabarin A, Terzolo M, Tsagarakis S \& Dekkers OM. Management of adrenal incidentalomas: European Society of Endocrinology Clinical Practice Guideline in collaboration with the European Network for the Study of Adrenal Tumors. European Journal of Endocrinology 2016 175 G1-G34. (https://doi.org/10.1530/EJE-16-0467)

3 Nakamura Y, Maekawa T, Felizola SJ, Satoh F, Qi X, VelardeMiranda C, Plonczynski MW, Ise K, Kikuchi K, Rainey WE, et al. Adrenal CYP11B1/2 expression in primary aldosteronism: immunohistochemical analysis using novel monoclonal antibodies. Molecular and Cellular Endocrinology 2014392 73-79. (https://doi. org/10.1016/j.mce.2014.05.002)

4 Smith A, Gaba RC, Bui JT \& Minocha J. Management of renovascular hypertension. Techniques in Vascular and Interventional Radiology 2016 19 211-217. (https://doi.org/10.1053/j.tvir.2016.06.006)

5 Anderson JL, Halperin JL, Albert NM, Bozkurt B, Brindis RG, Curtis LH, DeMets D, Guyton RA, Hochman JS, Kovacs RJ, et al. Management of patients with peripheral artery disease (compilation of 2005 and 2011 ACCF/AHA guideline recommendations): a report of the American College of Cardiology Foundation/American Heart 
Association Task Force on Practice Guidelines. Circulation 2013127 1425-1443. (https://doi.org/10.1161/CIR.0b013e31828b82aa)

6 Lee SY \& Lau H. Effectiveness of unilateral nephrectomy for renal hypertension in adults. Asian Journal of Surgery 200831 185-190. (https://doi.org/10.1016/S1015-9584(08)60083-4)

7 Tsunoda K, Abe K, Yamada M, Kato T, Yaoita H, Taguma Y, Goto Y $\&$ Ioridani N. A case of primary aldosteronism associated with renal artery stenosis and preclinical Cushing's syndrome. Hypertension Research 200831 1669-1675. (https://doi.org/10.1291/hypres.31.1669)

8 Debillon E, Velayoudom-Cephise FL, Salenave S, Caron P, Chaffanjon P, Wagner T, Massoutier M, Lambert B, Benoit M, Young J, et al. Unilateral adrenalectomy as a first-line treatment of Cushing's syndrome in patients with primary bilateral macronodular adrenal hyperplasia. Journal of Clinical Endocrinology and Metabolism 2015100 4417-4424. (https://doi.org/10.1210/jc.2015-2662)
9 Nishimoto K, Nakagawa K, Li D, Kosaka T, Oya M, Mikami S, Shibata H, Itoh H, Mitani F, Yamazaki T, et al. Adrenocortical zonation in humans under normal and pathological conditions. Journal of Clinical Endocrinology and Metabolism 201095 2296-2305. (https://doi.org/10.1210/jc.2009-2010)

10 Omata K, Anand SK, Hovelson DH, Liu CJ, Yamazaki Y, Nakamura Y, Ito S, Satoh F, Sasano H, Rainey WE, et al. Aldosterone-producing cell clusters frequently harbor somatic mutations and accumulate with age in normal adrenals. Journal of the Endocrine Society 20171 787-799. (https://doi.org/10.1210/js.2017-00134)

11 Sugiura Y, Takeo E, Shimma S, Yokota M, Higashi T, Seki T, Mizuno Y, Oya M, Kosaka T, Omura M, et al. Aldosterone and 18-oxocortisol coaccumulation in aldosterone-producing lesions. Hypertension 201872 1345-1354. (https://doi.org/10.1161/ HYPERTENSIONAHA.118.11243)

Received in final form 16 May 2020

Accepted 15 June 2020 